\title{
A Collaborative Study to Establish the Second Korean National Reference Standard for Snake Venom
}

\author{
Kiwon Han', Kikyung Jung ', Hokyung Oh', Hojin Song ${ }^{1,2}$, Sangmi Park ${ }^{1,2}$, Ji-Hye Kim ', Garam Min', \\ Byung-Hwa Lee ${ }^{3}$, Hyun-sik Nam ${ }^{4}$, Yang Jin Kim ${ }^{5}$, Manabu Ato ${ }^{6}$, Jayoung Jeong' and Chiyoung Ahn ${ }^{7}$ \\ ${ }^{1}$ Blood Products Division, National Institute of Food and Drug Safety Evaluation, Ministry of Food and Drug Safety, \\ Cheongju, Korea \\ ${ }^{2}$ Department of Manufacturing Pharmacy, Chungbuk National University College of Pharmacy, Cheongju, Korea \\ ${ }^{3}$ Quality Control Team, Korea Vaccine Co., Ltd., Ansan, Korea \\ ${ }^{4}$ New Drug Development Center, Osong Medical Innovation Foundation, Cheongju, Korea \\ ${ }^{5}$ Department of Statistics, Sookmyung Women's University College of Science, Seoul, Korea \\ ${ }^{6}$ Department of Immunology, National Institute of Infectious Diseases, Tokyo, Japan \\ ${ }^{7}$ Advanced Therapy Products Research Division, National Institute of Food and Drug Safety Evaluation, Ministry of \\ Food and Drug Safety, Cheongju, Korea
}

\begin{abstract}
In 2015, a candidate for the second national reference standard (NRS) of Gloydius snake venom was produced to replace the first NRS of Gloydius snake venom. In the present study, the potencies of the candidate were determined by a collaborative study, and the qualification of the candidate was estimated. The potencies of the candidate were determined by measuring the murine lethal titers and lapine hemorrhagic titers of venom against the regional working reference standard (RWRS) for antivenom using the methods described in the previous report for the first NRS of Gloydius snake venom. Three Korean facilities contributed data from a total of 30 independent assays. Subsequently, two foreign national control research laboratories contributed to this collaborative study. The results were calculated using the Reed-Muench method for lethality and determined using a mixed-effects model for hemorrhage. The general common potencies of the lethal and hemorrhagic titers were obtained from the results of the 30 tests performed at three Korean facilities. The results are expressed in micrograms for 1 test dose (TD) with a $95 \%$ confidence interval as follows: a lethal titer of $90.13 \mu \mathrm{g} / \mathrm{TD}(95 \%$ confidence interval $=87.39 \sim$ $92.86 \mu \mathrm{g})$ and a hemorrhagic titer of $10.80 \mu \mathrm{g} / \mathrm{TD}(95 \%$ confidence interval $=10.46 \sim 11.14 \mu \mathrm{g})$. In addition, the candidate preparation showed good quality evaluation according to the results of the quality estimation of the candidate and is judged to be suitable to serve as the Korean NRS for snake venom. In conclusion, the second NRS of Gloydius snake venom was established in this study and will be used for national quality control, including a national lot release test of Korean antivenom products.
\end{abstract}

Key words: National reference standard, Collaborative study, Gloydius snake venom, Lethal titer, Hemorrhagic titer

\footnotetext{
Correspondence to: Jayoung Jeong, Blood Products Division, National Institute of Food and Drug Safety Evaluation, Ministry of Food and Drug Safety, Osong Health Technology Administration Complex 187, Osongsaengmyeong-2-ro, Heungdeok-gu, Cheongju 28159, Korea

E-mail: 0jjy@korea.kr

Chiyoung Ahn, Advanced Therapy Products Research Division, National Institute of Food and Drug Safety Evaluation, Ministry of Food and Drug Safety, Osong Health Technology Administration Complex 187, Osongsaengmyeong-2-ro, Heungdeok-gu, Cheongju 28159, Korea

E-mail: cahn@korea.kr
}

This is an Open-Access article distributed under the terms of the Creative Commons Attribution Non-Commercial License (http:// creativecommons.org/licenses/by-nc/3.0) which permits unrestricted non-commercial use, distribution, and reproduction in any medium, provided the original work is properly cited. 


\section{INTRODUCTION}

The genus Gloydius (Serpentes: Crotalinae) was proposed to be redefined into Gloydius spp. from Agkistrodon spp. in 1997 (1). It is known that 3 venomous Gloydius snakes inhabit Korea: Gloydius brevicaudus, Gloydius intermedius, and Gloydius ussuriensis (2). Gloydius snakebite causes lethality by hemolysis and acts on capillaries or venules to cause hemorrhage, inducing hemorrhagic swelling and necrosis of the lesion area (3). These symptoms are caused by Gloydius snake venom, which has lethal and hemorrhagic activities (4). An antivenom product has been used in Korea for the treatment of Gloydius snakebites. Tests for quality control of the antivenom product have been performed according to the Korean minimum requirements using the reference standard for Gloydius snake venom and antivenom (5). In 2004, a previous collaborative study for the establishment of the regional working reference standard (RWRS) of Gloydius antivenom (code no. 011201) was conducted in Japan, China, and Korea (6). Since the RWRS of Gloydius antivenom was established in 2006, it has been used in the quality control of antivenom products. Meanwhile, the national reference standard (NRS) of Gloydius snake venom, which was manufactured and established for the first time in 2004 (7), has served as the reference standard for quality control testing of antivenom products. The assigned potency of the RWRS of Gloydius antivenom consists of the anti-lethal and anti-hemorrhagic titers, which offer neutralizing activities against the Gloydius snake venom reference standard. Furthermore, the NRS of Gloydius snake venom has lethal and hemorrhagic titers of $89.30 \mu \mathrm{g}$ and $7.91 \mu \mathrm{g}$, respectively, which are inversely determined against the RWRS of Gloydius antivenom (6,7). In 2015, a second candidate for the NRS of Gloydius snake venom originating from the venom of G. brevicaudus siniticus was newly produced to be used for national quality control because of a shortage in supply of the first NRS of Gloydius snake venom.

In the present study, the characteristics of the potency of the second candidate for the NRS of Gloydius snake venom were determined in a manner similar to that of the first NRS of Gloydius snake venom that had previously been established (7) through a collaborative study. The qualities of the candidate were estimated to judge whether it would be suitable for use as the Korean NRS for snake venom. Three Korean facilities (1 national control laboratory, 1 manufacturer and 1 medical research laboratory) contributed to this collaborative study. Two foreign national control laboratories (in Japan and Indonesia) subsequently participated in the collaborative study and compared results (See ACKNOWLEDGMENTS).

\section{MATERIALS AND METHODS}

Production of the second candidate for the NRS of Gloydius snake venom. The second candidate for the NRS of Gloydius snake venom (SV1507-1, 1,563 vials) was lyophilized at Korea Vaccine Co., Ltd (Ansan, Korea). The final bulk of Gloydius snake venom was supplied by Shanghai Serum Biological Technology (SSBT) (Zhejiang, China) by an identical process and using the same formulation of the first NRS of Gloydius snake venom in 2004; it contained $20 \mathrm{mg} \mathrm{G.} \mathrm{brevicaudus} \mathrm{siniticus} \mathrm{venom,}$ $8.5 \mathrm{mg} \mathrm{NaCl}, 10 \mathrm{mg}$ sucrose, $20 \mathrm{mg}$ human serum albumin, and Tris- $\mathrm{HCl}$ buffer $(0.01 \mathrm{M}, \mathrm{pH} 8.0)$ per $1 \mathrm{~mL}$ vial (8). Pilot studies were conducted to find the most suitable conditions for lyophilization of the snake venom. Korea Vaccine Co., Ltd. performed several preliminary tests, including an immunodiffusion assay and immuno-electrophoresis to evaluate the immunological characteristics associated with the snake venom (8).

Animals. The ICR strain of specific pathogen free (SPF) mice (female, $16 \pm 1 \mathrm{~g}$ ) were used for the determination of the snake venom titer associated with lethality in Korea and Indonesia. For the determination of the snake venom titer associated with hemorrhage, the New Zealand strain of the SPF rabbit (2 rabbits per 1 test; female, $2.0 \pm 0.2 \mathrm{~kg}$ ) was used in Korea. In Japan, the strain of mice for the lethal tests was Scl:ddY, and the Japanese white strain of rabbits was used for the hemorrhagic tests on the condition of equal weight in Korea. Four mice per group were used for the determination of the lethal titer (1 test dose). Ten mice per group were used for the determination of the lethal toxicity of the candidate for the second NRS of Gloydius snake venom, and 6 mice served as negative controls. All animal tests were approved by the NIFDS Ethics Committee on Animal Use (1601MFDS05 ) and conducted according to the Korean animal testing guidelines. Animals were housed in an SPF facility at 20 $24^{\circ} \mathrm{C}$ and a relative humidity of $55 \pm 10 \%$ with $15 \sim 20$ air changes/hour and $12 \mathrm{hr}$ light/darkness cycles. All the animals were acclimatized for one week prior to testing.

Determination of the lethal titer (1 test dose) and lethal toxicity. The lethal titer (1 test dose; $1 \mathrm{TD})$ of the candidate for the second NRS of Gloydius snake venom against the anti-lethal activity of Gloydius antivenom was determined at the National Institute of Food and Drug Safety Evaluation (NIFDS, Cheongju, Korea), Korea Vaccine Co., Ltd., Osong Medical Innovation Foundation (KBIO, Cheongju, Korea), National Institute of Infectious Diseases (NIID, Tokyo, Japan) and National Quality Control Laboratory of Drug and Food (NQCLDF, Central Jakarta, Indonesia) using the RWRS of Gloydius antivenom (code no. 011201, anti-lethal titer: 32,000 U/vial) using the test methods prescribed in Korea (7). The candidate was dissolved in $0.017 \mathrm{M}$ phosphate buffered sodium chloride solution containing $0.2 \mathrm{w} / \mathrm{v} \%$ gelatin $(\mathrm{pH} 7.0)$ (GPBS) to make a solution of $1,200 \mu \mathrm{g} / \mathrm{mL}$ and then serially diluted with GPBS such that $1 \mathrm{~mL}$ of each dilution contained 960, 
Table 1. Composition of assay mixtures $(\mathrm{mL})$ for the $1 \mathrm{TD}$ of lethal and hemorrhagic titer determination

\begin{tabular}{llcrrrr}
\hline \hline & Preparation & 1 & 2 & 3 & 4 & 5 \\
\hline Venom $^{*}$ & $1,200 \mu \mathrm{g} / \mathrm{mL}$ (Lethal titer) or $200 \mu \mathrm{g} / \mathrm{mL}$ (Hemorrhagic titer) & 1.000 & 0.800 & 0.640 & 0.510 & 0.410 \\
GPBS & & 0.000 & 0.200 & 0.360 & 0.490 & 0.590 \\
Antivenom & $100 \mathrm{U} / \mathrm{mL}$ (Lethal titer) or $10 \mathrm{U} / \mathrm{mL}$ (Hemorrhagic titer) & 1.000 & 1.000 & 1.000 & 1.000 & 1.000 \\
\hline
\end{tabular}

${ }^{*} 0.017 \mathrm{~mol} / \mathrm{L}$ phosphate buffered sodium chloride solution containing $0.2 \mathrm{w} / \mathrm{v} \%$ gelatin $(\mathrm{pH} 7.0)$.

Table 2. Composition of assay mixtures $(\mathrm{mL})$ for venom lethal toxicity and minimal hemorrhagic dose (MHD)

\begin{tabular}{llccccc}
\hline & Preparation & 1 & 2 & 3 & 4 & 5 \\
\hline Venom & $800 \mu \mathrm{g} / \mathrm{mL}$ (Lethal toxicity) or $20 \mu \mathrm{g} / \mathrm{mL}$ (MHD) & 4.000 & 2.000 & 1.000 & 0.500 & 0.250 \\
GPBS & & 0.000 & 2.000 & 3.000 & 3.500 & 3.750 \\
\hline
\end{tabular}

$768,614.4$, or $491.5 \mu \mathrm{g}$ of snake venom (Table 1). The RWRS of Gloydius antivenom was reconstituted and diluted in GPBS to a concentration of $100 \mathrm{U} / \mathrm{mL}$ (9). For neutralization, aliquots of $1 \mathrm{~mL}$ that appropriately diluted the candidate (Table 1) were mixed with $1 \mathrm{~mL}$ of the diluted antivenom and kept at room temperature for $1 \mathrm{hr}$. The mice were injected intravenously with $0.2 \mathrm{~mL}$ of each mixture.

The lethal toxicity of the candidate for the second NRS of Gloydius snake venom was determined at NIFDS, Korea Vaccine Co., Ltd., KBIO, and NIID. The toxicity was determined without the neutralization using the RWRS of Gloydius antivenom. The candidate was dissolved in 0.017 M GPBS ( $\mathrm{pH} 7.0$ ) to make a solution of $800 \mu \mathrm{g} / \mathrm{mL}$ and serially diluted with GPBS such that $1 \mathrm{~mL}$ of each dilution contained $400,200,100$, or $50 \mu \mathrm{g}$ of snake venom (Table 2). The mice were injected intravenously with 0.1 $\mathrm{mL}$ of each dilution. The lethal titer (1 TD) and lethal toxicity expressed in terms of the number of $50 \%$ lethal doses $\left(\mathrm{LD}_{50}\right)$ of the candidate was calculated using the ReedMuench method (10). Every mouse from all groups used for the determination of the lethal toxicity was necropsied and observed by NIFDS for gross lesions.

Determination of the hemorrhagic titer (1 test dose) and minimal hemorrhagic dose (MHD). The hemorrhagic titer (1 TD) of the candidate for the second NRS of Gloydius snake venom was calculated against the antihemorrhagic activity of the RWRS of Gloydius antivenom (code no. 011201, anti-hemorrhagic titer: 36,000 U/vial) at NIFDS, Korea Vaccine Co., Ltd., KBIO, and NIID using the Korea method (7).

The snake venom candidate was dissolved in GPBS to a concentration of $200 \mu \mathrm{g} / \mathrm{mL}$ and serially diluted with GPBS such that $1 \mathrm{~mL}$ of the dilution contained 160, 128, 102.4 , or $81.92 \mu \mathrm{g}$ (Table 1). The regional reference for the antivenom was reconstituted and diluted into GPBS to a final concentration of $10 \mathrm{U} / \mathrm{mL}$ (9). Aliquots of $1 \mathrm{~mL}$ of the appropriately diluted snake venom candidate were mixed with $1 \mathrm{~mL}$ of antivenom and kept at room temperature for $1 \mathrm{hr}$ (Table 1). Subsequently, aliquots of $0.2 \mathrm{~mL}$ of these mixtures were injected intradermally into each of the shaved backs of two rabbits for each mixture.

The MHD of the candidate for the second NRS of Gloydius snake venom was determined at NIFDS, Korea Vaccine Co., Ltd., KBIO, and NIID using the Korea method (7). The snake venom candidate was dissolved in GPBS to a concentration of $20.0 \mu \mathrm{g} / \mathrm{mL}$ and serially diluted with GPBS such that $1 \mathrm{~mL}$ of the dilution contained 10.0, 5.0, or $2.5 \mu \mathrm{g}$ (Table 2). Each dilution of $0.1 \mathrm{~mL}$ was injected into the dermal area on the shaved backs of two rabbits. After $24 \mathrm{hr}$, the rabbits were euthanized, and the back skin was removed using a surgical instrument. The hemorrhagic spots were measured from the inner side of the skin

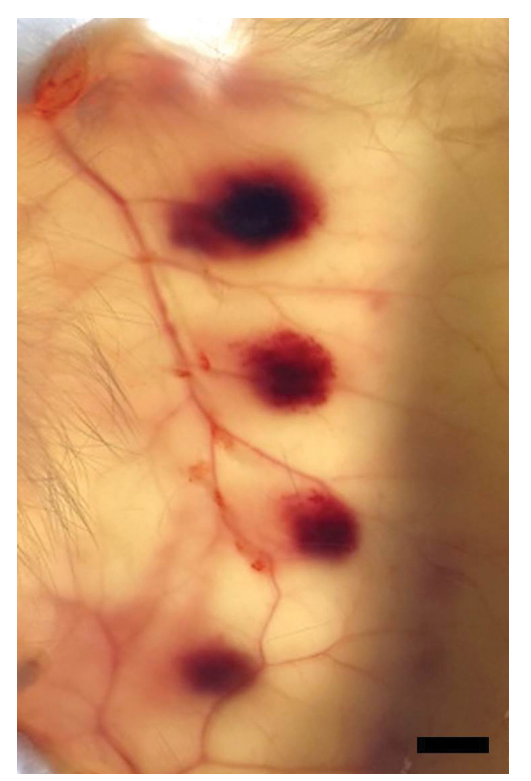

Fig. 1. Hemorrhagic spots for the MHD test results are shown. Back skin of euthanized rabbits was excised, and the hemorrhagic spots were measured from the inner side of the skin using a light box. The hemorrhagic spots resulted from intradermal injections of snake venom $(2.0 \mu \mathrm{g} / 0.1 \mathrm{~mL}, 1.0 \mu \mathrm{g} / 0.1$ $\mathrm{mL}, 0.5 \mu \mathrm{g} / 0.1 \mathrm{~mL}$, and $0.25 \mu \mathrm{g} / 0.1 \mathrm{~mL}$ ), from top to bottom, respectively. Bar, $10 \mathrm{~mm}$. 
using Vernier calipers (Fig. 1). The hemorrhagic titers (1 TD) and MHD of the snake venom candidate were expressed in terms of the size of the hemorrhagic spots (average cross-diameter of $10 \mathrm{~mm}$ ). The results were calculated using a mixed-effects model (8).

Estimation of the quality of the lyophilized snake venom candidate. The candidate was subjected to quality control tests including identification testing, physical appearance testing, sterility testing, water determination testing, mass variation testing, foreign insoluble matter testing, and leakage testing to ensure the quality of the lyophilized venom candidate as a Korean commercial antivenom product at Korea Vaccine Co., Ltd. All the tests were examined three times using each of the 10 vials containing the candidate preparation. These test methods were conducted according to the procedures prescribed in the Korean minimum requirements (5).

\section{RESULTS}

Determination of the lethal titer (1 test dose) and lethal toxicity. The $1 \mathrm{TD}$ of the lethal titers was $87.51 \mu \mathrm{g}$ (95\% confidence interval: 81.49 93.53 $\mu \mathrm{g}$ ) at NIFDS, $90.66 \mu \mathrm{g}(85.69 \sim 95.64 \mu \mathrm{g})$ at $\mathrm{KBIO}, 92.21 \mu \mathrm{g}(89.67 \sim$ $94.75 \mu \mathrm{g})$ at Korea Vaccine Co., Ltd., $99.30 \mu \mathrm{g}(88.95 \sim$ $109.65 \mu \mathrm{g})$ at NIID, and 92.81 $\mathrm{g}$ (87.04 98.59 $\mu \mathrm{g})$ at NQCLDF as shown in Table 3. Intra-laboratory variability was calculated as coefficients of variation $(\mathrm{CVs})$ and are also shown in Table 3. In all five laboratories, the CVs were approximately $10 \%(11.10 \%, 8.86 \%, 4.44 \%, 11.89 \%$, and $7.10 \%$, respectively). The general common potency determined in collaboration with the results of the three Korean facilities was $90.13 \mu \mathrm{g}$. This means that approximately $90.13 \mu \mathrm{g}$ of the venom is needed to induce the result of $\mathrm{LD}_{50}$ in the mice of the group injected intravenously with $0.2 \mathrm{~mL}$ of mixed solution neutralized with 10 $\mathrm{U} / \mathrm{mL}$ of antivenom.

The lethal toxicity is shown in Table 4 . The results were $27.50 \mu \mathrm{g}(95 \%$ confidence interval: $25.97 \sim 29.03 \mu \mathrm{g})$ at NIFDS, $27.50 \mu \mathrm{g}(25.97 \sim 29.03 \mu \mathrm{g})$ at KBIO, $28.28 \mu \mathrm{g}$ at Korea Vaccine Co., Ltd., and $27.97 \mu \mathrm{g}(27.33 \sim 28.60 \mu \mathrm{g})$ at NIID. Almost all of the mice intravenously injected with the candidate for the second NRS of Gloydius snake venom in the respective groups exhibited a similar degree of pulmonary lesions (edema and congestion). The mice in group 1, in which $0.1 \mathrm{~mL}$ of solution containing $80 \mu \mathrm{g}$ of snake venom was injected, showed severe gross lung lesions in almost all the lung lobes. The mice in group 2, in which $0.1 \mathrm{~mL}$ of solution containing $40 \mu \mathrm{g}$ of snake venom was injected, showed severe-to-moderate gross lung lesions (Fig. 2A, 2B, 2C). The mice in groups 3 ( $20 \mu \mathrm{g}$ of snake venom) and $4(10 \mu \mathrm{g}$ of snake venom) showed moderate-to-mild or mild gross lung lesions. The mice in group 5 ( $5 \mu \mathrm{g}$ of snake venom) showed mild or no gross lung lesions. The other findings were epistaxis and tissue necrosis in the tail area in almost every mouse in groups 1 and 2 (Fig. 2D, 2E). No gross lesions were observed in the mice that were used for negative controls throughout the experiment.

Determination of the hemorrhagic titer (1 test dose) and minimal hemorrhagic dose (MHD). The $1 \mathrm{TD}$ of the hemorrhagic titers was $10.02 \mu \mathrm{g}(95 \%$ confidence

Table 3. Results of collaborative study for the 1 TD of lethal titer and hemorrhagic titer determination

\begin{tabular}{|c|c|c|c|c|c|c|c|c|c|}
\hline \multirow{2}{*}{ Test } & \multicolumn{5}{|c|}{1 TD of Lethal titer $(\mu \mathrm{g})^{\pi}$} & \multicolumn{4}{|c|}{1 TD of Hemorrhagic titer $(\mu \mathrm{g})^{\top}$} \\
\hline & $1^{*}$ & $2^{\dagger}$ & $3^{*}$ & $4^{\S}$ & $5^{\|}$ & $1^{*}$ & $2^{\dagger}$ & $3^{*}$ & $4^{\S}$ \\
\hline 1 & 94.20 & 103.77 & 94.20 & 107.92 & 85.86 & 8.50 & 12.18 & 11.28 & 11.08 \\
\hline 2 & 78.58 & 94.20 & 94.20 & 107.92 & 102.65 & 8.87 & 10.99 & 11.44 & 8.99 \\
\hline 3 & 86.37 & 86.37 & 96.00 & 107.92 & 96.00 & 10.13 & 10.48 & 11.33 & 9.14 \\
\hline 4 & 68.37 & 94.20 & 96.00 & 86.37 & 89.78 & 8.67 & 10.81 & 11.26 & 9.69 \\
\hline 5 & 78.58 & 78.58 & 94.20 & 86.37 & 89.78 & 9.41 & 10.70 & 10.78 & 10.11 \\
\hline 6 & 94.20 & 94.35 & 86.37 & & & 10.87 & 12.23 & 11.40 & \\
\hline 7 & 86.37 & 94.20 & 86.37 & & & 11.36 & 11.19 & 11.84 & \\
\hline 8 & 94.20 & 96.00 & 86.37 & & & 11.19 & 10.89 & 11.81 & \\
\hline 9 & 98.23 & 86.37 & 94.20 & & & 10.22 & 10.65 & 9.73 & \\
\hline 10 & 96.00 & 78.58 & 94.20 & & & 10.94 & 11.38 & 11.38 & \\
\hline Common potency (\%) & 87.51 & 90.66 & 92.21 & 99.30 & 92.81 & 10.02 & 11.15 & 11.23 & 9.80 \\
\hline \multirow[t]{2}{*}{$95 \%$ confidence interval } & 81.49 & 85.69 & 89.67 & 88.95 & 87.04 & 9.34 & 10.77 & 10.85 & 9.06 \\
\hline & $\sim 93.53$ & $\sim 95.64$ & $\sim 94.75$ & $\sim 109.65$ & $\sim 98.59$ & $\sim 10.69$ & $\sim 11.53$ & $\sim 11.60$ & $\sim 10.54$ \\
\hline Coefficients of variation & 11.10 & 8.86 & 4.44 & 11.89 & 7.10 & 10.84 & 5.51 & 5.37 & 8.59 \\
\hline General common potency & 90.13 & & & - & - & 10.80 & & & - \\
\hline
\end{tabular}

${ }^{*}$ National Institute of Food and Drug Safety Evaluation (NIFDS), Cheongju, Korea. ${ }^{\dagger}$ Osong Medical Innovation Foundation (KBIO), Cheongju, Korea. ${ }^{\ddagger}$ Koreavaccine co., Ansan, Korea. ${ }^{5}$ National Institute of Infectious Diseases (NIID), Tokyo, Japan. "National Quality Control Laboratory of Drug and Food (NQCLDF), Central Jakarta, Indonesia. "Titers are expressed as $\mu \mathrm{g}$ per animal. 
Table 4. Results of collaborative study for lethal toxicity and minimal hemorrhagic dose (MHD)

\begin{tabular}{lrrrrrrrr}
\hline \hline \multirow{2}{*}{ Test } & \multicolumn{3}{c}{ Lethal toxicity $(\mu \mathrm{g})^{\|}$} & \multicolumn{3}{c}{ Minimal hemorrhagic dose (MHD) $(\mu \mathrm{g})^{\|}$} \\
\cline { 2 - 9 } & $1^{*}$ & $2^{\dagger}$ & $3^{\star}$ & $4^{\S}$ & $1^{*}$ & $2^{\dagger}$ & $3^{\ddagger}$ & $4^{\S}$ \\
\hline 1 & 28.28 & 28.28 & 28.28 & 27.32 & 0.64 & 1.11 & 0.64 & 0.29 \\
2 & 25.94 & 25.94 & 28.28 & 28.28 & 1.48 & 1.90 & 0.48 & 0.45 \\
3 & 28.28 & 28.28 & 28.28 & 28.30 & 0.82 & 0.91 & 0.79 & 0.39 \\
Common potency & 27.50 & 27.50 & 28.28 & 27.97 & 0.98 & 1.31 & 0.64 & 0.38 \\
$95 \%$ confidence interval & 25.97 & 25.97 & - & 27.33 & 0.48 & 0.71 & 0.46 & 0.28 \\
& 29.03 & 29.03 & - & -28.60 & $\sim 1.48$ & $\sim 1.90$ & $\sim 0.81$ & $\sim 0.47$ \\
Coefficients of variation (\%) & 4.92 & 4.91 & - & 2.00 & 44.72 & 40.33 & 24.21 & 21.48 \\
\hline
\end{tabular}

${ }^{*}$ National Institute of Food and Drug Safety Evaluation (NIFDS), Cheongju, Korea. ${ }^{\dagger}$ Osong Medical Innovation Foundation (KBIO), Cheongju, Korea. ${ }^{\ddagger}$ Koreavaccine co., Ansan, Korea. ${ }^{5}$ National Institute of Infectious Diseases (NIID), Tokyo, Japan. " Titers are expressed as $\mu g$ per animal.

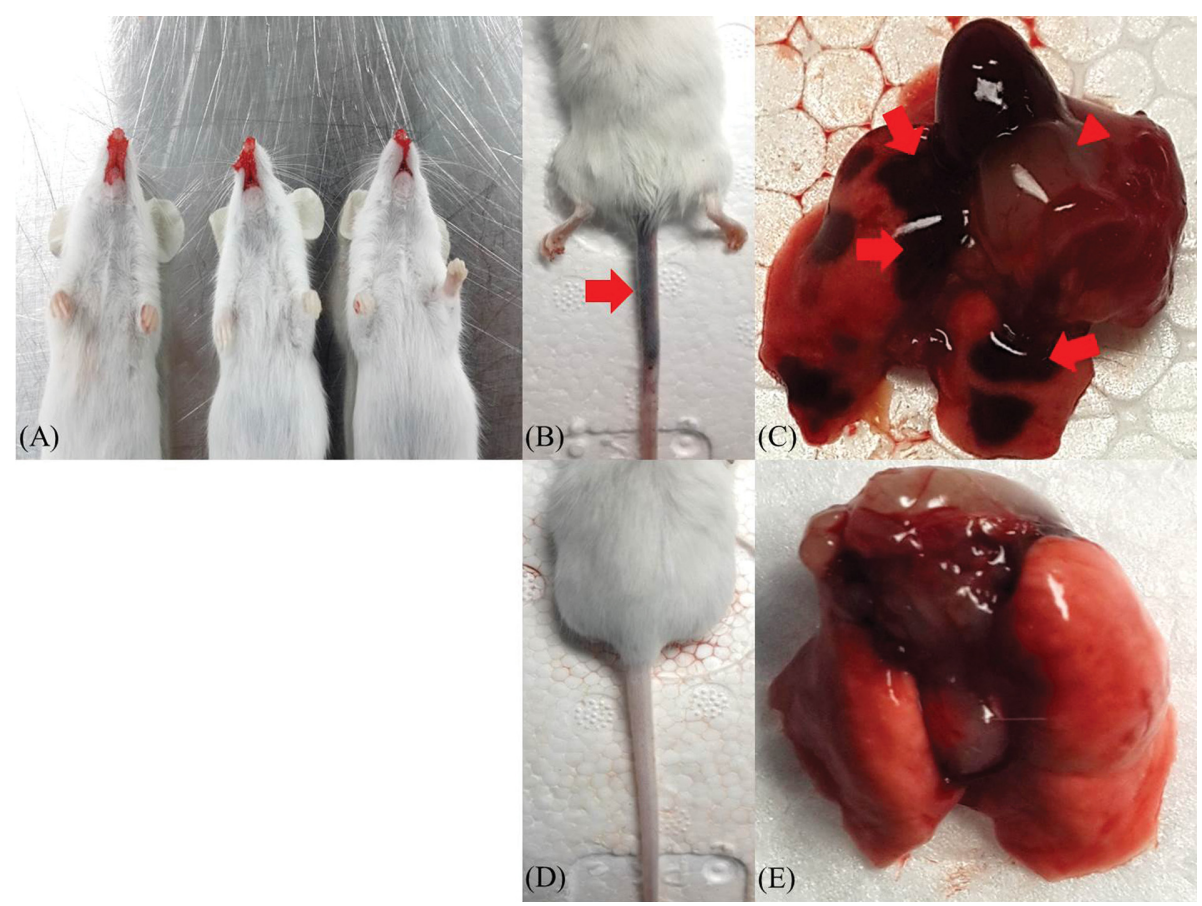

Fig. 2. Gross lesions in the mice intravenously injected with $0.1 \mathrm{~mL}$ of a solution containing $40 \mu \mathrm{g}$ of snake venom; (A) epistaxis, (B) tissue necrosis (red arrow), (C) pulmonary edema (red arrow head) and congestion (red arrow), (D) and (E) normal tissue of mice in the negative control group.

interval: $9.34 \sim 10.69 \mu \mathrm{g})$ at NIFDS, $11.15 \mu \mathrm{g}(10.77 \sim 11.53$ $\mu \mathrm{g})$ at $\mathrm{KBIO}, 11.23 \mu \mathrm{g}(10.85 \sim 11.60 \mu \mathrm{g})$ at Korea Vaccine Co., Ltd., and $9.80 \mu \mathrm{g}(9.06 \sim 10.54 \mu \mathrm{g})$ at NIID (Table 3). The CVs in the four laboratories were $10.84 \%, 5.51 \%$, $5.37 \%$, and $8.59 \%$, respectively. The general common potency of the hemorrhagic titer determined from the results of the thirty tests performed at NIFDS, KBIO, and Korea Vaccine Co., Ltd. was $10.80 \mu \mathrm{g}$. This means that approximately $10.80 \mu \mathrm{g}$ of the venom is needed to induce the size of the hemorrhagic spot (average cross-diameter of $10 \mathrm{~mm}$ ) in rabbits injected intradermally with $0.2 \mathrm{~mL}$ of mixed solution neutralized with the $1 \mathrm{U} / \mathrm{mL}$ of antivenom. The results of MHD were $0.98 \mu \mathrm{g}(95 \%$ confidence interval: $0.48 \sim 1.48 \mu \mathrm{g})$ at NIFDS, $1.31 \mu \mathrm{g}(0.71 \sim 1.90 \mu \mathrm{g})$ at
KBIO, $0.64 \mu \mathrm{g}(0.46 \sim 0.81 \mu \mathrm{g})$ at Korea Vaccine Co., Ltd., and $0.38 \mu \mathrm{g}(0.28 \sim 0.47 \mu \mathrm{g})$ at NIID (Table 4). One of the MHD results is shown in Fig. 1. The hemorrhagic spots induced by intradermal injection from top to bottom with the solution of $0.1 \mathrm{~mL}$ containing snake venom (in concentrations of $2.0 \mu \mathrm{g}, 1.0 \mu \mathrm{g}, 0.5 \mu \mathrm{g}$, and $0.25 \mu \mathrm{g}$ ) were of varying sizes depending on the snake venom concentration.

Estimation of the quality of the lyophilized snake venom candidate. The results of the quality control tests performed at Korea Vaccine Co., Ltd. are shown in Table 5. In all the test items, the quality of the snake venom candidate was verified to meet quality control standards. In the sterility test, there was no evidence of micro- 
Table 5. Results of the estimation of quality of the lyophilized venom candidate

\begin{tabular}{|c|c|c|c|c|c|}
\hline Test items & Specification & Test 1 & Test 2 & Test 3 & Test result \\
\hline Identification & Detect precipitation line & Pass & Pass & Pass & Pass \\
\hline Appearance & White or Lemon Yellow powder & Pass & Pass & Pass & Pass \\
\hline Sterility & No observed microorganism & Pass & Pass & Pass & Pass \\
\hline $\begin{array}{l}\text { Moisture content } \\
\text { (Water determination) }\end{array}$ & $<3.0 \%$ & $1.36 \%$ & $1.34 \%$ & $1.38 \%$ & $1.36 \%$ \\
\hline $\begin{array}{l}\text { Uniformity } \\
\text { (Mass variation test) }\end{array}$ & $<10 \%$ & $6.3 \%$ & $5.8 \%$ & $6.3 \%$ & $6.1 \%$ \\
\hline Foreign Insoluble particle matter & $\begin{array}{l}\text { No foreign matter in visual } \\
\text { (Optical particle counting: Over } 10 \mu \mathrm{g} \leq 6,000 \text { ea./container) }\end{array}$ & Pass & Pass & Pass & Pass \\
\hline Leakage & $\Delta p<3.82$ & 2.85 & 3.03 & 2.90 & 2.93 \\
\hline
\end{tabular}

bial growth. The results of the water determination test, mass variation test and foreign insoluble particle matter test fulfilled the requirements regarding each specification.

\section{DISCUSSION}

The World Health Organization (WHO) antivenom guidelines recommend that national reference standards or regional working reference standards of antivenoms or snake venoms associated with the antivenoms should be established by individual nations or regions (11). The reason for this recommendation is because there are no common international standards from the WHO in the antivenom fields due to the existence of considerable variation among the different snake species.

In 2015, the second candidate for the NRS of Gloydius snake venom was produced by the manufacturer using a certified manufacturing method. In the quality assessment, all test items obtained satisfactory results regarding the specifications, which meant that the snake venom candidate had quality characteristics similar to commercial antivenom products. The real-time stability test was performed at NIFDS where the snake venom candidate vials in triplicate were maintained at $-20^{\circ} \mathrm{C}$ for $0,3,6,9$, and 12 months. The control limits for monitoring the stability were set up in the range of $80 \sim 120 \%$ of the general common potencies, complying with the quality standard of other blood products (12). The lower control limits (LCL) of $1 \mathrm{TD}$ of the lethal titers were $72.10 \mu \mathrm{g}$ and $8.64 \mu \mathrm{g}$ in $1 \mathrm{TD}$ of the hemorrhagic titers. The upper control limits (UCL) were $108.15 \mu \mathrm{g}$ and $12.96 \mu \mathrm{g}$ in the lethal and hemorrhagic titers of $1 \mathrm{TD}$, respectively. The results of real-time stability were in the range of $80 \sim 120 \%$ of the control limits, and there were no significant differences among the $1 \mathrm{TD}$ of lethal and hemorrhagic titers of the candidates stored at $-20^{\circ} \mathrm{C}$ for 12 months (data not shown). These results indicated that there was no statistically significant loss of the potencies of the candidates according to storage time. The time interval and design of the continuous real-time stability test were according to the
International Conference on Harmonization of Technical Requirements for Registration of Pharmaceuticals for Human Use Guidelines (13). The clinical features of envenoming animals injected with the Gloydius snake venom were death secondary to hemorrhaging of the internal organs in the mice and circular ecchymoses in the skin of the rabbits. These observations were consistent with the previous study about the characterization of the snake venom of Agkistrodon spp. (Gloydius spp.) (4). However, the results were quite different in in the $1 \mathrm{TD}$ of the lethal titer and the MHD from Korea and Japan. There is no clear explanation for the significant difference among the results. It is assumed that there was a difference in the response to the injected snake venom or the assay mixture of snake venom and antivenom. The different responses may have occurred because of differences in the strain even within the same species of animals used for the tests in Korea and Japan. Therefore, further discussions are needed regarding the same test conditions including animals to be used and the number of tests to be performed among all the participants. Additionally, decisions should be made regarding whether the results would be included when there were results with a significant difference even though the tests were performed under the same conditions.

Unfortunately, the titers of the snake venom candidate were calculated similar to primary reference standards but not calibrated against the first NRS of Gloydius snake venom because of the shortage of the quantity for this collaborative study. However, the discriminating capacity of the present study was greatly enhanced by assessing the injected animals over a large number of tests. When the titers of 1 TD of the second NRS of Gloydius snake venom were compared with those of the first NRS of Gloydius snake venom, it revealed that the titers of the former (the first NRS) were higher than those of the latter (the second NRS). It means that the higher concentrations of the second NRS of Gloydius snake venom would be needed to produce the same results (i.e., the $\mathrm{LD}_{50}$ in the mouse groups and the size of the $10-\mathrm{mm}$ hemorrhagic spot in the rabbits) when mixed with the $10 \mathrm{U} / \mathrm{mL}$ or $1 \mathrm{U} / \mathrm{mL}$ of the 
RWRS of the Gloydius antivenom in the potency test.

This study aimed to establish the potencies of the second NRS of Gloydius snake venom. Gloydius snake venom is used in routine quality control tests by the NIFDS and manufacturers of the antivenom products. Approximately 50 vials per year are used for different purposes, including routine quality control tests and lot-release tests of antivenom products by manufacturers and the NIFDS as well as real-time stability tests in the designed periods at the NIFDS. The NIFDS will receive approximately 1,000 vials of the second NRS of Gloydius snake venom, which is sufficient to last as long as the potencies are maintained without observation of a significant decrease in their titers. In addition, the candidate preparation showed good quality evaluation according to the results of the estimation of the quality of the candidate and real-time stability tests. In conclusion, the candidate was judged to be suitable to serve as the second Korean NRS for Gloydius snake venom.

\section{ACKNOWLEDGMENTS}

The authors acknowledge Sun Hwa Choi and Young-Jin Lee (Quality Control Team, Korea Vaccine Co., Ltd., Korea), Nony Vilaning Tyas, Kusmiaty, and Atiek Supardiati (Biological Product Division, National Quality Control Laboratory of Drug and Food, National Agency of Drug and Food Control, The Requblic of Indonesia) for participating the collaboratory study.

\section{CONFLICT OF INTEREST}

The author's research was supported by a grant from the scientific research program (16201MFDS201) and a grantin-aid (MFDS 093-2100-2131-300) at the National Institute of Food Drug Safety Evaluation of the Ministry of Food and Drug Safety, Republic of Korea.

Received January 15, 2018; Revised April 3, 2018; Accepted April 16, 2018

\section{REFERENCES}

1. Wuster, W., Golay, P. and Warrell, D. (1997) Synopsis of recent developments in venomous snake systematics. Toxicon, 35, 319-340.

2. Lim, H., Kang, H.G. and Kim, K.H. (2013) Antivenom for snake bite in Korea. J. Korean Med. Assoc., 56, 1091-1103.

3. Eble, J.A. (2010) Matrix biology meets toxinology. Matrix Biol., 29, 239-247.

4. Omori, T., Iwanaga, S. and Suzuki, T. (1964) The relationship between the hemorrhagic and lethal of Japanese mamushi (Agkistrodon halys blomhoffi) venom. Toxicon, 2, 1-4.

5. Korea Food and Drug Administration (1993) Minimum Requirement for Biological Products (in Korean).

6. Fukuda, T., Iwaki, M., Hong, S.H., Oh, H.J., Wei, Z., Morokuma, K., Ohkuma, K., Dianliang, L., Arakawa, Y. and Takahashi M. (2006) Standardization of regional reference for mamushi (Gloydius blomhoffii) antivenom in Japan, Korea, and China. Jpn. J. Infect. Dis., 59, 20-24.

7. Yoo, S.H., Lee, N.K., Kim, S.N., Hong, C.M., Lee, K.H., Shin, I.S., Lim, J.H., Kim, Y.L., Ahn, M.J. and Park, S. (2004) Establishment of venom standard for potency test of Agkistrodon antivenom. The Annual Report of KFDA, 8-1, 1031-1036.

8. Oh, H., Shin, J., Ato, M., Ma, X., Williams, D., Han, K., Kim, Y.J., Kang, H.G., Jung, K., Hanada, K., Ochiai, M., Hung, P.V., Park, S. and Ahn, C. (2017) The First Meeting of the National Control Laboratories for Vaccines and Biologicals in the Western Pacific in 2016. Osong Public Health Res. Perspect., 8, 91-103.

9. Kondo, H., Kondo, S., Sadahiro, S., Yamauchi, K. and Murata, R. (1971) Standardazation of trimeresurus flavoviridis (Habu) antivenin. J. Med. Sci. Biol., 24, 323-327.

10. Reed, L.J. and Muench, H. (1938) A simple method of estimating fifty percent endpoints. Am. J. Epidemiol., 27, 493497.

11. World Health Organization (2010) WHO guidelines for the production control and regulation of snake antivenom immunoglobulins. World Health Organization, Geneva. [cited 2017 Jan 1]. Available from: http://www.who.int/bloodproducts/snake_antivenoms/snakeantivenomguide/en/.

12. Lee, N., Seo, J.S., Kim, J.O. and Ban, S.J. (2017) Establishment of the 2nd Korean national biological reference standard for blood coagulation factor VIII:C concentrate. Biologicals, 47, 59-63.

13. ICH (1995) Quality of biotechnological products: stability testing of biotechnological/products: stability testing of biotechnological/biological products. Q5C in International Conference on Harmonization of Technical Requirements for Registration of Pharmaceuticals for Human Use. 